\title{
Optimal Trajectory Generation in Ocean Flows
}

\author{
Tamer Inanc, Shawn C. Shadden and Jerrold E. Marsden
}

\begin{abstract}
In this paper it is shown that Lagrangian Coherent Structures (LCS) are useful in determining near optimal trajectories for autonomous underwater gliders in a dynamic ocean environment. This opens the opportunity for optimal path planning of autonomous underwater vehicles by studying the global flow geometry via dynamical systems methods. Optimal glider paths were computed for a 2 -dimensional kinematic model of an end-point glider problem. Numerical solutions to the optimal control problem were obtained using Nonlinear Trajectory Generation (NTG) software. The resulting solution is compared to corresponding results on LCS obtained using the Direct Lyapunov Exponent method. The velocity data used for these computations was obtained from measurements taken in August, 2000, by HF-Radar stations located around Monterey Bay, CA.
\end{abstract}

\section{INTRODUCTION}

In this paper we propose a method for determining near optimal trajectories in the ocean for a class of Autonomous Underwater Vehicles (AUVs) known as gliders. AUVs are becoming increasingly popular for collecting scientific data in the ocean. For example, they played an important role in the Office of Naval Research sponsored Autonomous Ocean Sampling Network II project (AOSN-II) [6], [1]. There is a growing body of literature on the use of AUVs for ocean sensing; for examples, see [2], [7], [19]. However, to the best of the authors' knowledge, there has not been much work done on optimization of AUV trajectories in the presence of ocean dynamics, which is the topic of the present paper.

Gliders offer an attractive means for gathering data in the ocean because they are low cost and highly sustainable. They are designed for high efficiency and can operate autonomously, which makes them good candidates for autonomous, large-scale ocean surveys. AOSN-II employed two types of gliders, the SLOCUM and the SPRAY [19].

The tradeoff for a glider's remarkable efficiency is a relatively low average speed for the vehicle. Typically, gliders move around $40(\mathrm{~cm} / \mathrm{s})$ relative to the ambient water. However, the ambient water can often move at speeds the same order of magnitude as the speed of the glider. For instance, in Monterey Bay, CA, which was the location for the AOSN-II experiment, surface currents average around

This work was partially supported by ONR/AOSN-II contract N0001402-1-0826 and AFOSR contract F49620-02-1-0176

T. Inanc is an Assistant Professor at the Department of Electrical and Computer Engineering, University of Louisville, W.S. Speed Bldg., Room 200, Louisville, KY 40292. t.inanc@ louisville.edu

S. C. Shadden (Corresponding Author) is a $\mathrm{PhD}$ student at the Department of Control and Dynamical Systems, California Institute of Technology, MC 107-81, Pasadena, CA 91125. shawn@cds.caltech.edu

J. E. Marsden is a Professor at the Department of Control and Dynamical Systems, California Institute of Technology, MC 107-81, Pasadena, CA 91125. marsden@cds.caltech.edu $20(\mathrm{~cm} / \mathrm{s})$, and are typically stronger outside the bay. Therefore it is advantageous, if not necessary, to make use of ocean currents to help propel the gliders around the ocean for sustainable missions. The idea of exploiting "natural dynamics" for vehicle transport has been used extensively in a number of research areas, such as in space mission design. For example, the natural dynamics of the 3- and 4body problem can be used to find efficient orbits for mission trajectories [9].

If the dynamics of the ocean are known a priori, an exhaustive optimization could be performed to numerically find an optimal trajectory each time a glider needed to move from one location to another. However the exact dynamics of the ocean are never known a priori, nor is it often practical to run such extensive computations.

The purpose of this paper is to propose a method for quickly determining near optimal glider trajectories based on approximate ocean current data. It will be shown that optimal trajectories computed using the Nonlinear Trajectory Generation (NTG) software correspond to Lagrangian Coherent Structures (LCS). These approximate solutions can then be used for either rough path planning or to initialize more detailed optimization computations. For realtime implementation, LCS must be computed from forecasts of ocean currents. However, it is reasonable to assume approximate ocean forecasts can be made [18] and LCS are robust to reasonable errors in the model forecasts [11].

The remainder of the paper is structured as follows: First we formulate the general optimal control problem. We discuss the utility of B-Splines and the Nonlinear Trajectory Generation software to compute numerical solutions of the optimal control problem. Next we solve the optimal control problem for the case of finding an optimal path between two fixed points in the ocean. We then motivate the use of LCS to help navigate the gliders and include a review of how LCS are computed. Finally we compare our solution to the optimal control problem with a corresponding LCS.

\section{Optimal Control Problem}

Consider a general dynamical (control) system

$$
\dot{\mathbf{x}}(t)=f(\mathbf{x}(t), \mathbf{u}(t))
$$

where $\mathbf{x}(t)$ is the state of the system and $\mathbf{u}(t)$ is the control input. For optimal control, we would like to choose $\mathbf{u}(t)$ such that some cost function is minimized and constraints are enforced. That is, given a cost function of the form

$$
\begin{aligned}
J= & \Phi_{0}\left(\mathbf{x}\left(t_{0}\right), \mathbf{u}\left(t_{0}\right), t_{0}\right)+\int_{t_{0}}^{t_{f}} L(\mathbf{x}(t), \mathbf{u}(t), t) d t \\
& +\Phi_{f}\left(\mathbf{x}\left(t_{f}\right), \mathbf{u}\left(t_{f}\right), t_{f}\right)
\end{aligned}
$$


we would like to choose $\mathbf{u}(t)$ for $t \in\left[t_{0}, t_{f}\right]$ which minimizes $J$ subject to (1) and constraints of the form

$$
\begin{array}{ll}
\text { Initial } & l b_{0} \leq \Psi_{0}\left(\mathbf{x}\left(t_{0}\right), \mathbf{u}\left(t_{0}\right), t_{0}\right) \leq u b_{0} \\
\text { Trajectory } l b_{t} \leq \Psi_{t}(\mathbf{x}(t), \mathbf{u}(t), t) & \leq u b_{t} \\
\text { Final } & l b_{f} \leq \Psi_{f}\left(\mathbf{x}\left(t_{f}\right), \mathbf{u}\left(t_{f}\right), t_{f}\right) \leq u b_{f}
\end{array}
$$

The cost function $J$ is composed of an initial condition cost, $\Phi_{0}(\cdot)$, an integral cost over the trajectory, $L(\cdot)$, and a final condition cost, $\Phi_{f}(\cdot)$. The constraints are similarly partitioned. $l b$ and $u b$ represent lower and upper bounds, respectively. Equations (2) and (3) are standard in optimal control, and are further explained in [5].

In most cases, the dynamics (1) and constraints (3) are too complicated for the minimization of (2) to be solved analytically, so numerical algorithms must be used to obtain solutions. To solve optimal control problems numerically, they are often transformed into nonlinear programming (NLP) problems. The software package, Nonlinear Trajectory Generation (NTG), is very useful for transforming the optimal control problem given in (2) to an NLP problem, see [14].

\section{A. Nonlinear Trajectory Generation}

If the dynamics, cost, and constraints are evaluated at discrete points in the interval $\left[t_{0}, t_{n}\right]$, it is possible to translate the optimization problem, defined by (1), (2) and (3), into the following NLP problem in $C_{j}$ :

$$
\min _{\vec{C} \in \mathbb{R}^{p}} F(\vec{C}) \text { subject to } L \leq G(\vec{C}) \leq U
$$

where $\vec{C}=\left[\begin{array}{lll}C_{1} & \cdots & C_{p}\end{array}\right]^{T} \cdot F(\vec{C})$ is our transformed cost function, and $G(\vec{C})$ is the transformation of the constraints, with $L$ and $U$ being the lower and upper bounds, respectively. The discrete points, $C_{i}$, at which cost and constraints are evaluated are known as collocation points.

The NTG software package, developed by Milam et. al. [14], is based on a combination of nonlinear control theory, spline theory, and sequential quadratic programming. NTG takes the optimal control problem formulation, characterization of trajectory space, and the set of collocation points, and transforms them into an NLP problem. It is then solved using NPSOL [8], a popular NLP solver, which uses Sequential Quadratic Programming (SQP) to obtain the solution.

\section{B. B-Splines}

To facilitate numerical computation, outputs of the optimization, along with the cost function and constraints given in (2) and (3) are expressed in terms of B-spline functions, see [4] for a detailed treatment of splines, and [3], [15], [16] for their use in optimal control problems.

B-Spline curves are constructed by joining Bézier curves with a prescribed level of continuity. The points at which the curves are joined are called breakpoints. The nondecreasing list of real numbers containing the breakpoints is the knot vector, $K=\left\{t_{0}, t_{1}, \cdots, t_{n}\right\}$, and $n$ is the number of intervals. The number of times a breakpoint occurs in a knot vector is called the multiplicity, $m_{i}$. The smoothness, $s_{i}$, of a breakpoint provides the level of continuity; a breakpoint is $\left(s_{i}-1\right)$ times continuously differentiable. The order, $r_{i}$, of each piecewise polynomial is $r_{i}=s_{i}+m_{i}$ for interior breakpoints. We will assume that the smoothness, $s_{i}$, and the multiplicity, $m_{i}$, are the same for all breakpoints.

A trajectory $\mathbf{x}(t)$ with prescribed smoothness $s$ and order $r$ can be written as

$$
x(t)=\sum_{j=1}^{p} B_{j, r}(t) C_{j} ; t_{0} \leq t \leq t_{n}
$$

where $p$ is defined by $p=n(r-s)+s$, which is the number of free parameters $C_{j}$ (coefficients of the B-Spline functions) that can be used to customize the trajectory. The functions $B_{j, r}(t)$ are B-Spline basis functions defined by

$$
B_{j, 1}(t)=\left\{\begin{array}{l}
1 \quad \text { if } t_{j} \leq t<t_{j+1} \\
0 \quad \text { otherwise }
\end{array}\right.
$$

and $B_{j, 1}=0$ if $t_{j}=t_{j+1}$. Higher order terms can be found using the Cox-de Boor recursion formula for $r>1$, see [4].

\section{Using Temporal Constraints with NTG}

While the NTG formulation allows any spatial constraint to be easily coded into the constraint set, including temporal constraints requires more care. The easiest way to solve this is to introduce time as a state variable in the optimization.

First define the new scaled time variable $\tau$ shown in (6), where $t$ represents the true time, or old time, and $T$ is the new state variable representing the unknown final time which will be optimized. In the setup of the optimization problem detailed in Sec. III, scaled time $\tau$ rather than true time $t$ is used:

$$
\tau=\frac{t}{T}
$$

After introducing the new state variable $T$, the cost and constraint functions given in (2) and (3) become

$$
\begin{aligned}
& J(\mathbf{x}, \mathbf{u}, T)= \Phi_{0}(\mathbf{x}(0), \mathbf{u}(0), T)+\Phi_{f}(\mathbf{x}(1), \mathbf{u}(1), T) \\
&+\int_{0}^{1} L(\mathbf{x}(\tau), \mathbf{u}(\tau), T) d \tau \\
& l b_{0} \leq \Psi_{0}(\mathbf{x}(0), \mathbf{u}(0), T) \leq u b_{0} \\
& l b_{f} \leq \Psi_{f}(\mathbf{x}(1), \mathbf{u}(1), T) \leq u b_{f} \\
& l b_{t} \leq \Psi_{t}(\mathbf{x}(\tau), \mathbf{u}(\tau), T) \leq u b_{t}
\end{aligned}
$$

Any additional temporal constraints may be expressed as a set of inequalities given by

$$
l b_{T} \leq \Psi_{T}(T) \leq u b_{T} \quad N_{T} \text { temporal constraints. }
$$




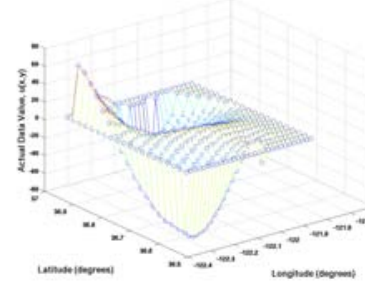

a) $\mathrm{u}(\mathrm{x}, \mathrm{y})$ Data, $t=1$

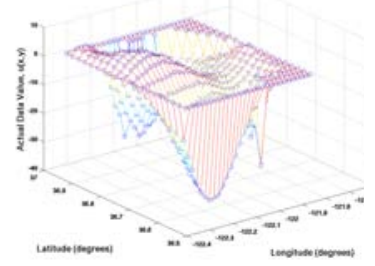

c) $\mathrm{u}(\mathrm{x}, \mathrm{y})$ Data, $t=4$

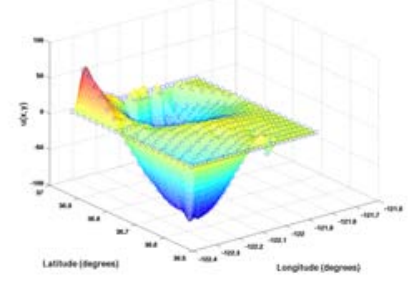

b) $\mathrm{u}(\mathrm{x}, \mathrm{y})$ Model, $t=1$

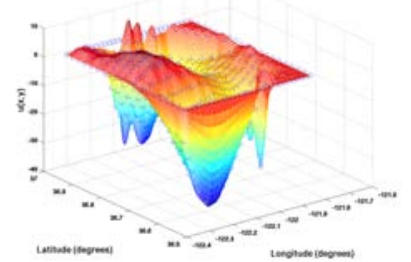

d) $\mathrm{u}(\mathrm{x}, \mathrm{y})$ Model, $t=4$

Fig. 1. The Ocean Current Data and B-Spline Models for Time $=1$ and 4 (hrs)

\section{Optimal Control Example}

Now that we have reviewed the formulation of the optimal control problem, let us consider a particular example. Consider the problem of finding an optimal glider trajectory between two fixed points in the ocean. Denote the starting point $\mathbf{x}_{0}$ and the end point $\mathbf{x}_{f}$. In particular, we will consider two points in Monterey Bay, CA, whose longitude/latitude coordinates are given by

$$
\begin{aligned}
& \mathbf{x}\left(t_{0}\right)=(-122.178(\mathrm{deg}), 36.8557(\mathrm{deg})) \\
& \mathbf{x}\left(t_{f}\right)=(-122.242(\mathrm{deg}), 36.6535(\mathrm{deg}))
\end{aligned}
$$

For the purposes of determining the glider trajectory, a 2-D kinematic model will be used:

$$
\begin{aligned}
& \dot{x}=V \cos \theta+u \\
& \dot{y}=V \sin \theta+v
\end{aligned}
$$

where $V$ is the speed of the vehicle, $\theta$ is direction of motion, and $u$ and $v$ are the components of the ocean currents in the $x$ - and $y$-direction ${ }^{1}$, respectively. These equations represent the equations of motion given in (1), with $V$ and $\theta$ being the control inputs. The pair $(u(x, y, t), v(x, y, t))$ is referred to as the (time-dependent) velocity field ${ }^{2}$.

The velocity field data was obtained from High Frequency Radar stations that measure surface currents in Monterey Bay, CA [17]. The data was processed by OpenBoundary Modal Analysis [13] to smooth the data and fill in missing data points.

In the NTG framework, the user needs to specify the following:

- Choice of outputs and their derivatives

\footnotetext{
${ }^{1}$ Coordinates are chosen such that the $x$-axis is in the direction of increasing longitude and the $y$-axis in the direction of increasing latitude.

${ }^{2}$ For this paper, we only consider 2-dimensional flow, even though the ocean is 3-dimensional. However, for most purposes, the z-component of the ocean is negligible.
}

- The cost and the constraints in terms of these outputs and their derivatives

- The regularity of the variables, placement of the knot points, order and regularity of the B-Splines, and collocation points for each output

Thus, NTG needs the derivatives of the velocity field with respect to the outputs. Numerically computing these derivatives directly from the velocity data sets can easily create convergence problems so it is best to use the tensor product B-Spline functions, allowing straightforward computation of derivatives.

The general B-Spline parameterizations for this example are given as:

$$
\begin{aligned}
& u(x, y)=\sum_{i=1}^{m} \sum_{j=1}^{n} B_{i, k_{u x}}(x) B_{j, k_{u y}}(y) a_{i j} \\
& v(x, y)=\sum_{i=1}^{p} \sum_{j=1}^{r} B_{i, k_{v x}}(x) B_{j, k_{v y}}(y) b_{i j}
\end{aligned}
$$

where $a_{i j}$ and $b_{i j}$ represent coefficients of the B-Spline functions for $u(x, y)$ and $v(x, y)$, respectively. $B_{i, k}$ and $B_{j, k}$ represent $\mathrm{B}-$ Spline basis functions for the $x$ - and $y$ direction, respectively. The order of the polynomials used were $k_{u x}=k_{u y}=k_{v x}=k_{v y}=4$ and the number of the coefficients were $m=p=32$ and $n=r=22$. Fig. 1 shows $\mathrm{u}(\mathrm{x}, \mathrm{y})$ from ocean current data and the $\mathrm{B}-$ Spline representations at times $t=1$ and $t=4$ hours.

The cost function for this problem is a weighted sum of a temporal cost and an energy cost as follows:

$$
\begin{aligned}
J & =W_{t} T \\
& +\int_{0}^{1} W_{u}\left(\left(\frac{\dot{x}}{T}-u\right)^{2}+\left(\frac{\dot{y}}{T}-v\right)^{2}\right) T d \tau
\end{aligned}
$$

where $\dot{x}=d x / d t$. Note that the $T$ terms in the integral are due to introducing time as a state variable in the NTG formulation. Integral bounds range from 0 to 1 from the re-scaling transformation. $W_{t}$ and $W_{u}$ represent the weighting on the total mission time and energy expenditure, respectively.

Constraint functions are given as:

- (Linear) Initial Constraints:

$$
\begin{gathered}
-122.1780-\epsilon(\mathrm{deg}) \leq x(0) \leq-122.1780+\epsilon(\mathrm{deg}) \\
36.8557-\epsilon(\mathrm{deg}) \leq y(0) \leq 36.8557+\epsilon(\mathrm{deg}) \\
0 \leq T \leq 48 \text { hours }
\end{gathered}
$$

- (Linear) Final Constraints:

$$
\begin{gathered}
-122.2420-\epsilon(\operatorname{deg}) \leq x(T) \leq-122.2420+\epsilon(\operatorname{deg}) \\
36.6535-\epsilon(\operatorname{deg}) \leq y(T) \leq 36.6535+\epsilon(\operatorname{deg})
\end{gathered}
$$

- (Nonlinear) Trajectory Constraint:

$$
1 \leq W_{v} \frac{1}{T^{2}}\left(\left(\frac{d x}{d \tau}\right)^{2}+\left(\frac{d y}{d \tau}\right)^{2}\right) \leq 1600
$$

where $\epsilon$ is a small positive number and $W_{v}$ represents the weighting on the velocity of the glider. 


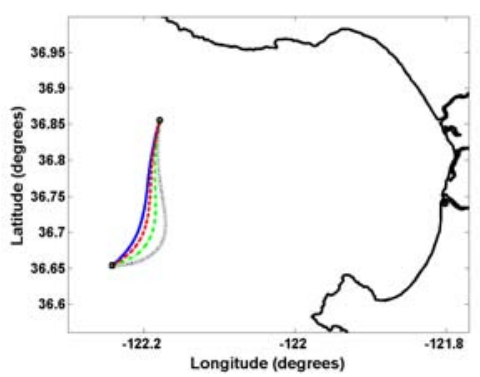

(a) Velocity Field

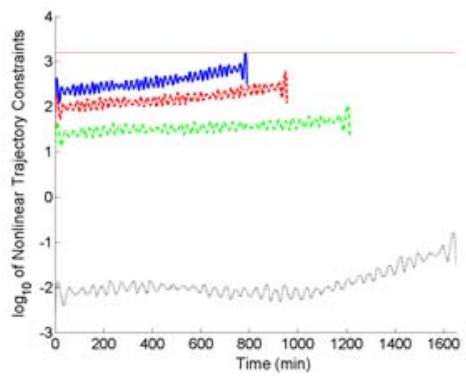

(b) Trajectories

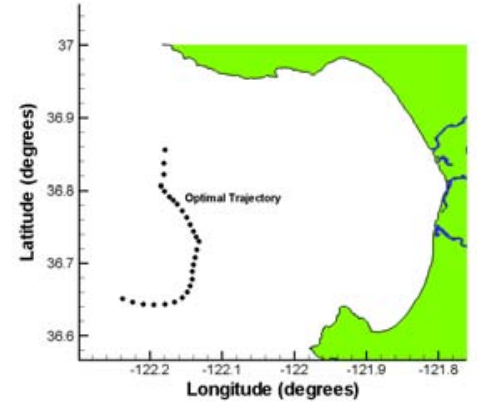

(c) DLE field

Fig. 2. Panel (a) shows four trajectories with varying emphasis on $W_{t}$ and $W_{u}$, the solid line is for $W_{t}>>W_{u}$ and the dotted line for $W_{t}<<W_{u}$. Panel (b) shows the trajectory constraints of the four trajectories shown in panel (a). Panel (c) shows the receding-horizon optimal trajectory.

The linear initial constraints serve to define the start position of the glider and initialize the time. Linear final constraints restrict the final destination point of the glider. The nonlinear trajectory constraint limits the velocity of the glider to a maximum relative velocity of $40(\mathrm{~cm} / \mathrm{s})$.

To understand how $W_{t}$ and $W_{u}$ affect optimal solutions, consider the differences between trajectories from heavily weighting energy as opposed to time. Fig. 2(a) compares trajectories which range from $W_{t}>>W_{u}$, in the solid line, to $W_{t}<<W_{u}$ in the dotted line. As expected, the trajectories are more direct when time is weighted more than energy. We see that weighting energy causes the trajectories to deviate from a straight line, which indicates the effect of ocean currents.

One can see an even more striking distinction between these trajectories by comparing the plots of their nonlinear constraint functions shown in Fig. 2(b). The constraint values are plotted on a log-scale and that the square-root of the nonlinear constraint gives the relative speed of each glider. Upon close inspection, one will notice that the minimum-time glider shown by the solid blue line moves on average about three times faster (relative to the water), and over a shorter path, than the glider represented by the green dash-dotted line. But remarkably, the total trajectory times between these two only vary by about $30 \%$. Therefore, one can decidedly conclude the utility of the currents in the energy-optimal solutions. This motivates the need for a systematic way to utilize the ocean currents for navigating the gliders. This is accomplished by the help of Lagrangian Coherent Structures (LCS) in the ocean. In Section IV we review how LCS are obtained from Direct Lyapunov Exponent (DLE) fields.

\section{A. Receding-Horizon Optimal Trajectory}

The parameterizations given by (11) do not incorporate the time dependence of the currents. To build in the time dependence of the velocity data into NTG, we assume the velocity fields are constant over hourly intervals. For example, at time $t=0$ an optimal trajectory from $\mathbf{x}\left(t_{0}\right)$ to $\mathbf{x}\left(t_{f}\right)$ is computed assuming the velocity field given at $t=0$ does not change in time. Denote this trajectory $\hat{\mathbf{x}}_{1}(s)$. Then another next trajectory, $\hat{\mathbf{x}}_{2}(s)$, is computed by letting the initial point be $\hat{\mathbf{x}}_{1}(1$ hour $)$ and keeping the same endpoint $\mathbf{x}\left(t_{f}\right)$ while assuming the velocity field given at $t=1$ hour is constant in time. Continuing, we let the optimal trajectory, $\mathbf{x}_{\mathrm{opt}}(s)$, be the concatenation:

$$
\mathbf{x}_{\mathrm{opt}}(s)= \begin{cases}\hat{\mathbf{x}}_{1}(s) & \text { for } 0 \leq s<1 \\ \hat{\mathbf{x}}_{2}(s) & \text { for } 1 \leq s<2 \\ \vdots & \\ \hat{\mathbf{x}}_{n}(s) & \text { for } T-1 \leq s \leq T\end{cases}
$$

where $\hat{\mathbf{x}}_{n}(T)$ satisfies the terminals constraints. We think of this as a receding-horizon approach, where we update the best estimate of the currents each hour, which is reasonable since the currents do not change significantly in one hour. Fig. 2(c) shows the complete optimal trajectory for this example, where $W_{u}$ is slightly larger than $W_{t}$.

\section{Lagrangian Coherent Structures}

In the sections above, we discussed the use of optimization for determining efficient trajectories for a glider in the ocean. Now let us discuss some dynamical systems concepts which will allow us to determine similar efficient routes.

General time-dependent flows can often be studied in terms of Lagrangian Coherent Structures (LCS) [10]. LCS can be thought of as material lines which act as separatrices, dividing the flow into dynamically distinct regions. LCS can be defined in a number of ways; below we recall a definition based on Direct Lyapunov Exponents [20].

\section{A. Direct Lyapunov Exponent Approach}

Consider a general time-dependent dynamical system described by the differential equation

$$
\dot{\mathbf{x}}=f(\mathbf{x}, t)
$$

Let $\mathbf{x}\left(\mathbf{x}_{0}, t_{0} ; t\right)$ denote the solution of (13). Define the strain tensor

$$
\Sigma\left(\mathbf{x}_{0}, t_{0} ; t\right)=\left[\frac{\partial \mathbf{x}\left(\mathbf{x}_{0}, t_{0} ; t\right)}{\partial \mathbf{x}_{0}}\right]^{T}\left[\frac{\partial \mathbf{x}\left(\mathbf{x}_{0}, t_{0} ; t\right)}{\partial \mathbf{x}_{0}}\right]
$$




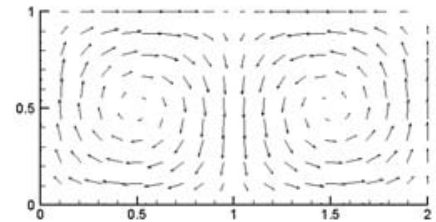

(a) Velocity Field, Double Gyre

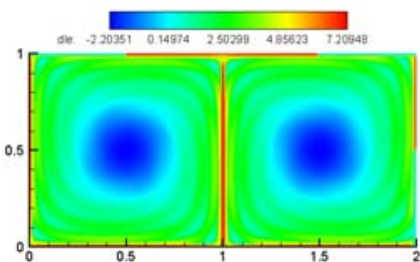

(b) DLE field, Double Gyre

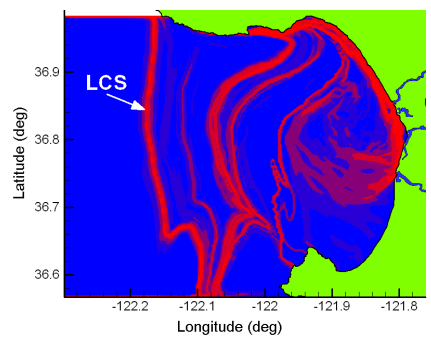

(c) DLE field, Monterey Bay

Fig. 3. Panel (a) shows the velocity field of the double gyre example. Panel (b) shows the DLE field of the double-gyre example; the red ridge of high DLE in the middle of the domain represents an LCS, which divides the flow into two distinct regions. Panel (c) shows a representative DLE field for Monterey Bay; areas of blue indicated low DLE, while the ridges of red indicated high DLE, which are Lagrangian Coherent Structures (LCS).

Let $\lambda_{t}\left(t_{0}, \mathbf{x}_{0}\right)$ denote the maximum eigenvalue of $\Sigma\left(\mathbf{x}_{0}, t_{0} ; t\right)$. The Direct Lyapunov Exponent (DLE) is defined as

$$
\sigma_{\Delta}\left(t_{0}, \mathbf{x}_{0}\right)=\frac{1}{2 \Delta} \ln \lambda_{t}\left(t_{0}, \mathbf{x}_{0}\right)
$$

where $\Delta=t-t_{0}$. The DLE characterizes the amount of stretching at a point $\mathbf{x}_{0}$ at time $t_{0}$.

If we fix the initial time $t_{0}$ then, with a slight abuse of notation, $\sigma_{\Delta}\left(t_{0}, \mathbf{x}_{0}\right)$ can be thought of as a scalar field, being a function of the spatial variable $\mathbf{x}_{0}$. For 2-dimensional flows, Lagrangian Coherent Structures are defined as ridges of the field $\sigma_{\Delta}\left(t_{0}, \mathbf{x}_{0}\right)$, see [20] for a precise definition.

As an example of these concepts, consider the "doublegyre", whose velocity field is shown in Fig. 3(a). The DLE field for this example is shown in Fig. 3(b) and we notice high DLE along the red line which divides the gyres. We refer to this line of high DLE as an LCS. This LCS should be expected since, from Fig. 3(a), two points which are close to each other, but on opposite sides of the line, will rapidly diverge when advected by the flow.

An example DLE field for Monterey Bay, CA, is shown in Fig. 3(c). It should be noted that for general timedependent flows, such as the ocean, LCS are typically timedependent curves. Therefore Fig. 3(c) should be interpreted as a snapshot of the time-dependent DLE field at a particular instance.

Again, LCS can be thought of as material lines, which act as separatrices. The intuition behind this for 2-dimensional, incompressible flow is that since there is high stretching about the LCS, then we must have a situation where particles on either side of the LCS are being advected into dynamically different regions of the flow, and to preserve continuity, particles on the LCS are advected along the structure (this reasoning can easily be justified by referring to "toy" example given in Figs. 3(a),(b)). Since LCS delineate the average motion of the flow, it is reasonable to assume that they might make efficient pathways. As shown in the next section, it turns out that this is indeed the case.

\section{COMPARISON OF RESUltS}

Now we are in a position to test the hypothesis that LCS in the ocean reveal efficient or near-optimal routes for glider transport. In Sec. III we had chosen boundary conditions (i.e. $\mathbf{x}\left(t_{0}\right)$ and $\mathbf{x}\left(t_{f}\right)$ ) near an LCS in Monterey Bay. In Fig. 4 we have superimposed instances of the trajectory given in Fig. 2(c) with the corresponding DLE field at that time. The figure should be thought of as snapshots of a movie which shows the progression of the LCS and the progression of the glider path together ${ }^{3}$. One can see that there is indeed a good correspondence between the optimal trajectory and the LCS.

This suggests something quite interesting: one can construct approximations to optimal paths by knowing the evolution of the LCS. It should be noted that although the initial location of the glider given in our example was near an LCS, this fortuity does not seem so contrived when one considers that LCS often correspond to geophysical fronts, such as temperature, which the gliders are meant to study [12]. Additionally, these LCS often represent separatrices, dividing regions of qualitatively distinct behavior. Therefore, gliders navigating along such dividing lines can easily switch to one or the other region with minimal control.

\section{CONClusions And Future Work}

In this paper we showed that LCS provide a good correspondence with optimal trajectories for autonomous underwater gliders in the ocean. The ability to navigate gliders efficiently is very important for sustainability and keeping maintenance and operational costs low. Therefore, analyzing the evolution of LCS can be quite important in planning paths for glider trajectories. While showing the existence of this correspondence is interesting, work is being done to develop systematic ways to exploit this relation in controlling gliders in the ocean in actual experiments, such as AOSN.

Some questions that will be studied in the near future are: Can computations of optimal trajectories be sped up by using information of LCS to initialize the optimization code? How does the trade-off between optimizing energy and optimizing time affect the utility of LCS for "optimal"

\footnotetext{
${ }^{3}$ This movie can be found at www.cds.caltech.edu/ shawn/animations/ACC05.html
} 


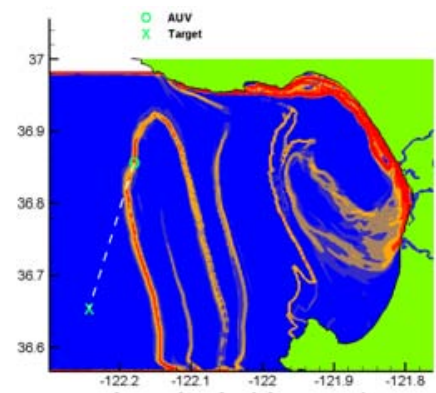

(a) Time $=0$ (hrs)

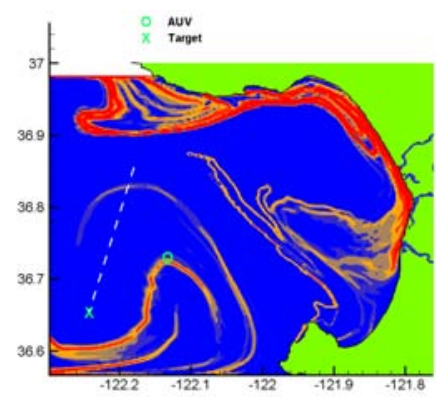

(d) Time $=15(\mathrm{hrs})$

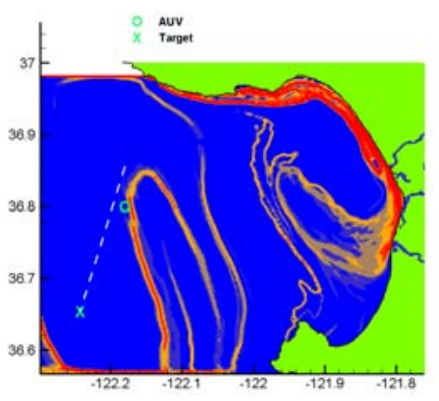

(b) Time $=5(\mathrm{hrs})$

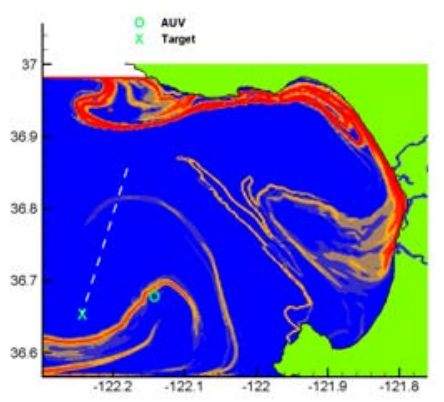

(e) Time $=20(\mathrm{hrs})$

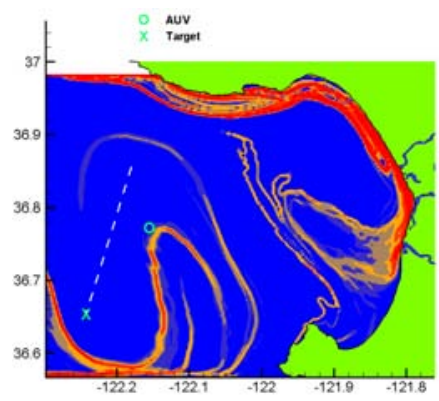

(c) Time $=10(\mathrm{hrs})$

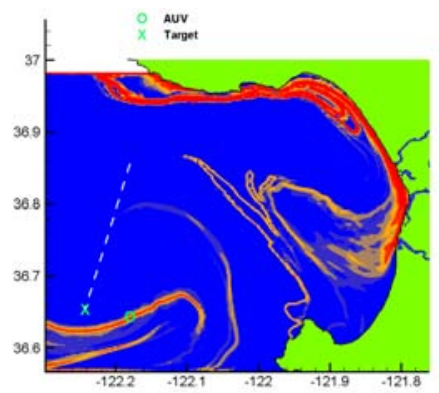

(f) Time $=25(\mathrm{hrs})$

Fig. 4. This figure shows the correspondence with the optimal trajectory shown in Fig. 2(c) and an LCS. Note that the 'O' in the figures near the LCS represents the location of the AUV while the ' $X$ ' represents the final target location. The movie version of these figures can be found at www.cds.caltech.edu/ shawn/animations/ACC05.html.

path planning? Also, in this paper we assumed a recedinghorizon approach to integrating the currents into the optimal control, however this integration should be incorporated continuously by extending the B-spline parameterizations in time (as well as space), which is something the authors are working on.

\section{ACKNOWLEDGEMENTS}

We would like to sincerely thank Raktim Bhattacharya, Francois Lekien, Richard Murray, and Naomi Leonard for their valuable input and support.

\section{REFERENCES}

[1] Autonomous Ocean Sampling Network Homepage, Edited September 02, 2003, http://www.mbari.org/aosn/default.htm.

[2] R. Bachmayer, N.E. Leonard, J. Graver, E. Fiorelli, P. Bhatta, and D. Paley "Underwater Gliders: Recent Developments and Future Applications", Proc. IEEE International Symposium on Underwater Technology (UT'04), Tapei, Taiwan, 2004.

[3] John T. Betts, Practical Methods for Optimal Control Using Nonlinear Programming, Society of Industrial and Applied Mathematics, 2001.

[4] Carl de Boor, A Practical Guide to Splines, Springer-Verlag, New York, 2001.

[5] A.E. Bryson Jr. and Y.C. Ho, Applied Optimal Control: Optimization, Estimation and Control, Taylor \& Francis, 1975.

[6] T. B. Curtin, J. G. Bellingham, J. Catipovic, and D. Webb, "Autonomous Oceanographic Sampling Networks", Oceanography, 6:8694, 1989.

[7] E. Fiorelli, N.E. Leonard, P. Bhatta, D. Paley, R. Bachmayer, and D.M. Fratantoni, "Multi-AUV Control and Adaptive Sampling in Monterey Bay", Proc. IEEE Autonomous Underwater Vehicles 2004: Workshop on Multiple AUV Operations (AUV04), Sebasco, ME, June 2004.
[8] P.E. Gill, W. Murray, M.A. Saunders, and M.H Wright, NPSOL nonlinear programming software. Stanford Business Software, Inc., 2680 Bayshore Parkway, Suite 304, Mountain View, CA 94043.

[9] G. Gomez, W.S. Koon, M.W. Lo, J.E. Marsden, J. Masdemont and S.D. Ross, "Invariant Manifolds, the Spatial Three-Body Problem and Space Mission Design", Proceedings of AIAA/AAS Astrodynamics Specialist Meeting, Quebec City, Quebec, Canada, 2001.

[10] G. Haller and G. Yuan. Lagrangian coherent structures and mixing in two-dimensional turbulence. Physica D, 147:352-370, 2000.

[11] G. Haller, "Lagrangian Coherent Structures from Approximate Velocity Data", Physics of Fluids, Vol 14, No 6, 1851-1861, 2002.

[12] F. Lekien, Time-Dependent Dynamical Systems and Geophysical Flows, PhD Thesis, California Institute of Technology, 2003.

[13] F. Lekien, C. Coulliette, R. Bank, J. Marsden, "Open-Boundary Modal Analysis: Interolation, Extrapolation and Filtering", Journal of Geophysical Research - Oceans, Vol. 109, No. C12, C12004 10.1029/2004JC002323, 2004.

[14] M.B. Milam, K Mushambi, and R.M. Murray, "A New Computational Approach to Real-Time Trajectory Generation for Constrained Mechanical Systems", Conference on Decision and Control, 2000.

[15] K. Misovec, T. Inanc, J. Wohletz and R.M. Murray, "Low-Observable Nonlinear Trajectory Generation for Unmanned Air Vehicles", 42nd IEEE Conference on Decision and Control, Maui, Hawaii, 9-12 December, 2003.

[16] C. P. Neuman and A. Sen, "A Suboptimal Control Algorithm for Constrained Problems using Cubic Splines", Automatica, v. 9, pg. 601-613, 1973.

[17] J.D. Paduan and L. K. Rosenfeld, "Remotely Sensed Surface Currents in Monterey Bay from Shore-Based HF Radar (CODAR)", $J$. Geophys. Res., 101, 20669-20686.

[18] A.R. Robinson, "Forecasting and Simulating Coastal Ocean Processes and Variabilities with the Harvard Ocean Prediction System" Coastal Ocean Prediction (C.N.K. Mooers, ed.), AGU Coastal and Estuarine Studies Series, American Geophysical Union, 77-100.

[19] D. L. Rudnick, R. E. Davis, C. C. Eriksen, D. M. Fratantoni, and M. J. Perry. "Underwater gliders for ocean research". In press, 2004.

[20] S.C. Shadden, F. Lekien, J.E. Marsden, "Definition and Properties of Lagrangian Coherent Structures", Submitted to Physica D. 